\title{
Afatinib ameliorates osteoclast differentiation and function through downregulation of RANK signaling pathways
}

\author{
Hye Jung Ihn ${ }^{1}$, Ju Ang Kim ${ }^{1}$, Yong Chul Bae ${ }^{2}$, Hong-In Shin ${ }^{1}$, Moon-Chang Baek ${ }^{3, *}$ E Eui Kyun Park ${ }^{1, *}$ \\ Departments of ${ }^{1}$ Oral Pathology and Regenerative Medicine, ${ }^{2}$ Anatomy and Neurobiology, School of Dentistry, Kyungpook National \\ University, Daegu 41940, ${ }^{3}$ Department of Molecular Medicine, CMRI, School of Medicine, Kyungpook National University, Daegu 41944, \\ Korea
}

\begin{abstract}
Non-small-cell lung cancer (NSCLC) is the third most common cancer that spreads to the bone, resulting in osteolytic lesions caused by hyperactivation of osteoclasts. Activating mutations in epidermal growth factor receptor-tyrosine kinase (EGF-TK) are frequently associated with NSCLC, and afatinib is a first-line therapeutic drug, irreversibly targeting EGF-TK. However, the effects of afatinib on osteoclast differentiation and activation as well as the underlying mechanism remain unclear. In this study, afatinib significantly suppressed receptor activator of nuclear factor $\kappa B$ (RANK) ligand (RANKL)-induced osteoclast formation in bone marrow macrophages (BMMs). Consistently, afatinib inhibited the expression of osteoclast marker genes, whereas, it upregulated the expression of negative modulator genes. The bone resorbing activity of osteoclasts was also abrogated by afatinib. In addition, afatinib significantly inhibited RANKL-mediated Akt/protein kinase B and c-Jun $\mathrm{N}$-terminal kinase phosphorylation. These results suggest that afatinib substantially suppresses osteoclastogenesis by downregulating RANK signaling pathways, and thus may reduce osteolysis after bone metastasis. [BMB Reports 2017; 50(3): 150-155]
\end{abstract}

\section{INTRODUCTION}

The bone undergoes a constant remodeling process to replenish and maintain bone volume, mineral density, and architecture. Balance between bone formation by osteoblasts and resorption by osteoclasts is critical for normal remodeling.

*Corresponding authors. Moon-Chang Baek, Tel: +82-53-420-4948; Fax: +82-53-420-4944; E-mail: mcbaek@knu.ac.kr, Eui Kyun Park, Tel: +82-53-420-4995; Fax: +82-53-428-4995; E-mail: epark@knu. ac.kr

https://doi.org/10.5483/BMBRep.2017.50.3.223

Received 21 December 2016, Revised 1 January 2017, Accepted 25 February 2017

Keywords: Afatinib, Bone resorption, Differentiation, Osteoclast, RANK signaling
Secretory factors including the RANK ligand (RANKL), macrophage colony-stimulating factor (M-CSF), and osteoprotegerin (OPG) are involved in a functional-coupling mechanism between osteoblasts and osteoclasts. RANKL and M-CSF secreted by osteoblasts promote the differentiation of preosteoclasts into osteoclasts $(1,2)$. OPG is a decoy receptor for RANKL, and thus, blocks the interaction between RANKL and RANK. The RANKL/OPG ratio is critical for controlling RANKL-induced osteoclast formation and activation $(3,4)$.

Bone volume and architecture can be altered by the pathological imbalance between bone formation and bone resorption. Metastatic tumor cells in the bone modulate the bone microenvironment and disrupt bone remodeling. Malignant cells secrete many growth factors that are involved directly or indirectly in osteoclast differentiation and activation, leading to osteolysis by increased bone resorption. These include RANKL, interleukin-1, interleukin-6, parathyroid hormone related protein (PTHrP), and macrophage inflammatory protein-1- $\alpha$ (5). Enhanced bone resorption, in turn, releases transforming growth factor- $\beta$ and insulin-like growth factor- 1 from the bone matrix, which stimulates PTHrP production and promotes tumor growth $(6,7)$.

Lung cancer, the third most common cancer to metastasize to the bone, is classified into two main groups: NSCLC and small cell lung cancer. NSCLC accounts for $80-85 \%$ of lung cancers; the common types include squamous cell carcinoma, large cell carcinoma, adenocarcinoma, and several other types. As NSCLC progresses, approximately $30-40 \%$ of patients develop bone metastasis (8). Bone metastasis has a significant morbidity burden, including osteolysis, bone pain, hypercalcaemia, fractures, spinal cord compression, and bone marrow infiltration (9).

Activating mutations in the epidermal growth factor receptor (EGFR) are largely associated with NSCLC; and treatment of EGF-mutation-positive NSCLC patients with reversible EGFR tyrosine kinase inhibitors (EGFR-TKIs), such as erlotinib and gefitinib, improve progression-free survival as compared with chemotherapy (10, 11). However, EGFR mutation-positive patients who respond to these EGFR-TKIs inevitably develop resistance after approximately 1 year of treatment (12). The second-generation irreversible EGFR-TKI, afatinib, has shown 
clinical efficacy in phase III trials in patients with NSCLC and head and neck squamous cell cancer. In 2013, afatinib was approved as the first-line treatment of EGFR mutation-positive NSCLC (13). However, whether afatinib can relieve the skeletal burden after bone metastasis remains unclear.

EGFR is known to be involved in the formation of osteoclasts. EGFR-deficient mice show delayed primary ossification due to defective osteoclast recruitment (14). RANKL-RANK binding induces an interaction with EGFR, which is required for osteoclast differentiation and survival (15), suggestive of an interaction between RANK and EGFR signaling pathways in osteoclast formation. Therefore, we hypothesized that in addition to its anticancer effects, afatinib may abolish osteoclast differentiation and functioning by downregulating RANK signaling pathways.

\section{RESULTS}

Afatinib suppresses RANKL-induced osteoclast differentiation To determine the effect of afatinib on RANKL-induced

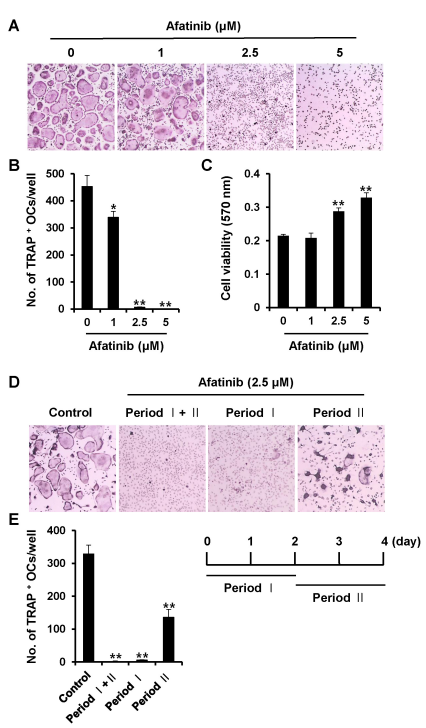

Fig. 1. Afatinib suppresses RANKL-induced osteoclast differentiation. (A) BMMs were cultured for 4 days with M-CSF $(10 \mathrm{ng} / \mathrm{ml})$ and RANKL $(20 \mathrm{ng} / \mathrm{ml})$ in the presence of $0,1,2.5$, or $5 \mu \mathrm{M}$ afatinib. Osteoclasts were stained with TRAP. (B) TRAP-positive multinucleated cells with $\geq 3$ nuclei were counted. ${ }^{*} P<0.05$ and $* * P$ $<0.01$ versus vehicle-treated control. (C) BMMs were cultured for 3 days with M-CSF $(10 \mathrm{ng} / \mathrm{ml})$ in the presence or absence of $1,2.5$, or $5 \mu \mathrm{M}$ afatinib. Cell viability was evaluated using the MTT assay. (D) BMMs were cultured with M-CSF $(10 \mathrm{ng} / \mathrm{ml})$ and RANKL $(20 \mathrm{ng} / \mathrm{ml})$, and afatinib $(2.5 \mu \mathrm{M})$ treatment was carried out from day 0 to day 4 (period $\mathrm{I}+\mathrm{II}$ ), from day 0 to day 2 (period I), and from day 3 to day 4 (period II). Osteoclast formation was assessed by TRAP staining. (E) TRAP-positive multinucleated cells with $\geq 3$ nuclei were counted. $* * P<0.01$ versus vehicle-treated control. osteoclast differentiation, BMMs were cultured with M-CSF (10 $\mathrm{ng} / \mathrm{ml})$ and RANKL $(20 \mathrm{ng} / \mathrm{ml})$ in the presence or absence of afatinib $(1,2.5$, and $5 \mu \mathrm{M})$. After 4 days, TRAP-positive MNCs were generated in response to M-CSF and RANKL. However, treatment with afatinib reduced osteoclast formation in a concentration-dependent manner (Fig. $1 \mathrm{~A}$ and 1B). Afatinib $(2.5 \mu \mathrm{M})$ significantly suppressed the formation of TRAPpositive MNCs (98.3\% inhibition) (Fig. 1B). To determine whether the inhibitory effects of afatinib were caused by cytotoxicity, the viability of osteoclast precursors was evaluated using the MTT assay. Afatinib (up to $5 \mu \mathrm{M}$ ) showed no cytotoxic effect on BMMs (Fig. 1C). We further evaluated the stage-specific effect of afatinib during osteoclast differentiation. Osteoclast formation was nearly abolished when afatinib was added from the beginning of culture to the time of pre-osteoclast formation (period I) (Fig. 1D and 1E). However, when afatinib was treated after pre-osteoclast formation (period II), TRAP-positive MNCs were still formed, but the number of TRAP-positive MNCs decreased by $58.7 \%$. In addition, the morphology of TRAP-positive MNCs was not

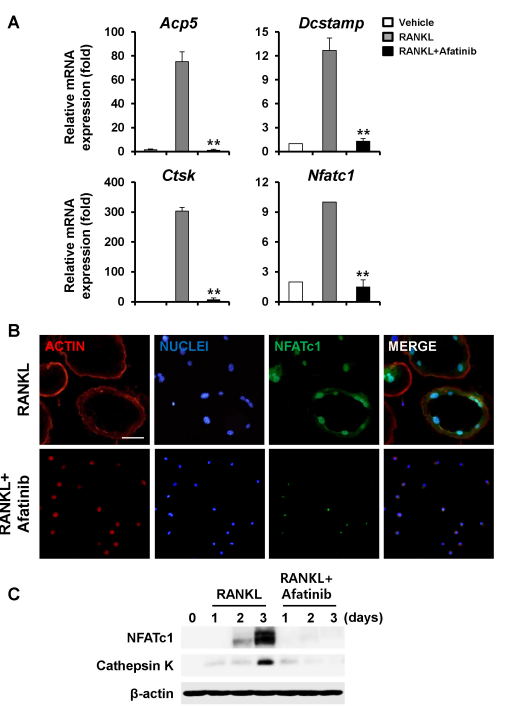

Fig. 2. Afatinib suppresses the expression of osteoclast markers and localization of NFATC1. (A) BMMs were cultured for 4 days with M-CSF $(10 \mathrm{ng} / \mathrm{ml})$ and RANKL $(20 \mathrm{ng} / \mathrm{ml})$ in the presence of $2.5 \mu \mathrm{M}$ afatinib. The mRNA expression of TRAP (Acp5), DCSTAMP (Dcstamp), cathepsin K (Ctsk), and NFATc1 (Nfatc1) was analyzed by real-time RT-PCR. ${ }^{* * P}<0.01$. (B) BMMs seeded onto glass coverslips were incubated for 4 days with M-CSF (10 $\mathrm{ng} / \mathrm{ml})$ and RANKL $(20 \mathrm{ng} / \mathrm{ml})$ in the presence or absence of afatinib $(2.5 \mu \mathrm{M})$. The cells were stained with anti-NFATc1 antibody, and actin rings and nuclei were stained with rhodamineconjugated phalloidin and DAPI, respectively. Fluorescent images were obtained. Magnification; 100X. Scale bar; $50 \mu \mathrm{m}$. (C) BMMs were cultured with M-CSF $(10 \mathrm{ng} / \mathrm{ml})$ and RANKL $(20 \mathrm{ng} / \mathrm{ml})$ in the presence or absence of $2.5 \mu \mathrm{M}$ afatinib for the indicated days. The cell lysates were analyzed by western blotting with antibodies against NFATc1 and cathepsin K. 
round or oval shaped, indicating that the actin ring structure had not properly formed (Fig. 1D). These results demonstrated that afatinib acts on both the formation of pre-osteoclast and proper morphology of mature osteoclasts.

\section{Afatinib downregulates the expression of osteoclast-specific markers}

To further elucidate the role of afatinib in osteoclastogenesis, the expression of osteoclast-specific markers was determined by real-time PCR (qPCR) and immunoblotting. BMMs stimulated with M-CSF and RANKL were treated with afatinib $(2.5 \mu \mathrm{M})$. As shown in Fig. 2A, RANKL significantly upregulated the expression levels of TRAP (Acp5), cathepsin $\mathrm{K}$ (Ctsk), DC-STAMP (Dcstamp), and NFATc1 (Nfatc1). Compared with RANKL-treated controls, the expression levels were significantly decreased by treatment with afatinib (Fig. 2A and 2C). Immunofluorescence analysis was performed to examine the expression level and nuclear translocation of NFATc1, a major transcription factor involved in regulating the differentiation of BMMs into osteoclasts. Afatinib treatment significantly decreased the expression level of RANKL-induced NFATc1 in the nucleus as well as the number of cells positive for nuclear NFATc1 (Fig. 2B). Based on the decreased level of Dcstamp, the formation of multinucleated giant cells was suppressed by treatment with afatinib (Fig. 2B). These data indicated that afatinib inhibits the expression of RANKL-induced genes involved in osteoclast differentiation and function.

A

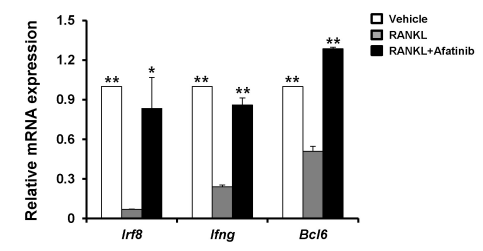

B

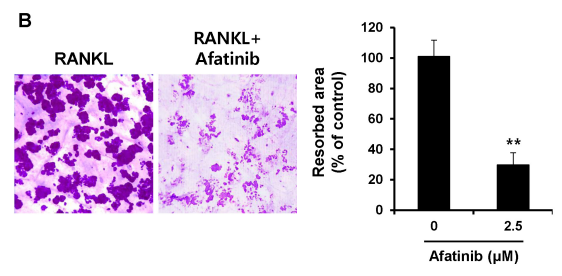

Fig. 3. Afatinib restores negative regulators for osteoclast differentiation and inhibits resorption pit formation. (A) BMMs were cultured for 4 days with M-CSF (10 ng/ml) and RANKL (20 $\mathrm{ng} / \mathrm{ml}$ ) in the presence of $2.5 \mu \mathrm{M}$ afatinib. The mRNA expression of Irf8, Ifng, and Bcl6 was analyzed by real-time RT-PCR. *P < 0.05 , and $* * P<0.01$ versus vehicle-treated control. (B) BMMs were plated onto bone slices and incubated with M-CSF (10 $\mathrm{ng} / \mathrm{ml})$ and RANKL $(20 \mathrm{ng} / \mathrm{ml})$ for 3 days to induce differentiation into osteoclasts. The cells were treated with or without afatinib $(2.5 \mu \mathrm{M})$ for an additional 2 days. Resorption pits were visualized by staining with hematoxylin. ${ }^{*} \mathrm{P}<0.01$ versus vehicle-treated control.

\section{Afatinib inhibits the suppression of negative mediators of RANKL-induced osteoclast differentiation}

During RANKL-induced osteoclast differentiation, RANK signaling downregulates the expression of interferon regulatory factor-8 (IRF8) and B-cell lymphoma 6 (Bcl6), which act as negative regulators of osteoclastogenesis $(16,17)$. To investigate the effect of afatinib on the negative mediators of osteoclast differentiation, we analyzed Irf8, Ifng, and Bcl6 transcript levels using qPCR. As shown in Fig. 3A, the inhibition of Irf8, Ifng, and Bcl6 mRNA expression mediated by RANKL was abrogated by treatment with afatinib, indicating that blocking the suppression of these negative molecules impaired osteoclast differentiation.

\section{Afatinib attenuates bone resorption activity}

Mature and active osteoclasts contain actin ring structures that create sealing zones between the cells and bone matrix during the resorption phase $(18,19)$. However, in the differentiation stage-specific experiment, osteoclast morphology appeared abnormal or immature (Fig. 1D). Therefore, we examined whether afatinib modulates osteoclast activity by using the resorption pit assay. BMMs were incubated on bone slices in osteoclastinducing medium for 3 days to generate osteoclast- like MNCs, and subsequently treated with afatinib or vehicle for an additional 2 days. As shown in Fig. 3B, afatinib markedly inhibited the formation of resorption pits, as compared to the positive control (70.5\% reduction). The result suggested that afatinib strongly suppresses the bone-resorbing activity of osteoclasts.

\section{Afatinib downregulates RANKL-induced phosphorylation of Akt/PKB and JNK}

After demonstrating that afatinib dramatically suppresses osteoclast differentiation and activation, we next evaluated whether afatinib inhibits RANK signaling pathways. Phospho-

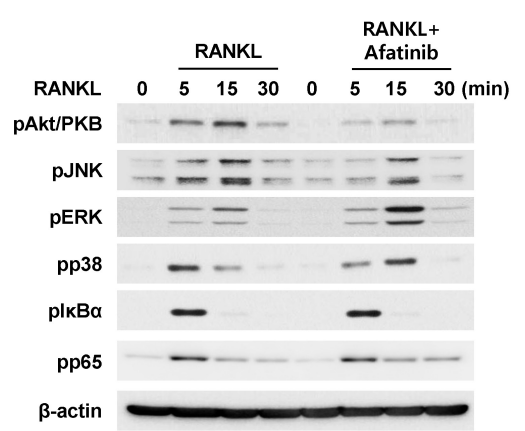

Fig. 4. Afatinib regulates RANK signaling pathways. BMMs were incubated in serum-free medium for $5 \mathrm{~h}$, and then pretreated with afatinib $(2.5 \mu \mathrm{M})$ or vehicle for $1 \mathrm{~h}$ before RANKL (50 $\mathrm{ng} / \mathrm{ml}$ ) stimulation for the indicated times. Phosphorylation of p38, Akt/PKB, ERK, JNK, I $\mathrm{\kappa B}$, and p65 was determined by western blotting using phospho-specific antibodies. Total $\beta$-actin was used as the loading control. 
rylation of Akt/PKB, c-Jun N-terminal kinase (JNK), p38, extracellular signal-related kinase (ERK), $I \kappa B$, and p65 was induced from $5 \mathrm{~min}$ after RANKL stimulation (Fig. 4). Pretreatment with afatinib decreased RANKL-induced Akt/PKB and JNK phosphorylation, whereas phosphorylation of p38, ERK, I $\mathrm{KB}$, and p65 was not downregulated (Fig. 4). Phosphorylation of $\mathrm{p} 38$ and ERK was somewhat activated at 15 min by afatinib treatment. These results suggested that afatinib inhibits the activation of $\mathrm{Akt} / \mathrm{PKB}$ and JNK, leading to suppression of RANKL-induced osteoclast differentiation.

\section{DISCUSSION}

Afatinib is the first-line treatment drug for NSCLC with EGFR mutations $(20,21)$. It irreversibly blocks homo- and heterodimeric ErbB receptors (EGFR/ErbB1, HER2/ErbB2, ErbB3, and ErbB4) $(22,23)$. Afatinib's reactive acrylamide group covalently and irreversibly binds to specific cysteine residues in the kinase domains of EGFR (773), HER2 (805), and HER4 (803), and inhibits auto- and transphosphorylation of the receptors (22-24). Our study results indicated that afatinib significantly inhibits osteoclast differentiation and activation. Afatinib treatment of BMMs incubated with RANKL and M-CSF significantly inhibited osteoclast differentiation (Fig. 1); in addition, osteoclast differentiation marker genes were strongly inhibited by afatinib (Fig. 2A). These results demonstrated that afatinib directly inhibits osteoclast differentiation. Because differentiating osteoclasts express EGFR, ErbB2, ErbB3, and ErbB4 (15), afatinib may target EGFR and other ErbB isoforms. These results are consistent with those of previous studies showing that reversible EGF-TKIs and shRNA targeting EGFR can inhibit osteoclast differentiation and survival (15).

The indirect action of EGFR in osteoclast differentiation has also been suggested. Gefitinib, a reversible EGFR-TKI, inhibits the expression of RANKL and M-CSF in human bone marrow stromal cells, and thus suppresses osteoclast differentiation (25). Erlotinib also inhibits osteolytic bone invasion of the human NSCLC cell line $\mathrm{NCl}-\mathrm{H} 292$ by downregulating RANKL in osteoblast/stromal cells (26). Therefore, EGFR-TKIs including afatinib, gefitinib, and erlotinib can directly or indirectly suppress osteoclast differentiation.

In this study, afatinib significantly induced negative regulators of osteoclast differentiation such as Irf8, Ifng, and Bcl6 (Fig. 3A). IRF8 can block NFATc1, the master transcription factor in osteoclast differentiation (17), and thus block the differentiation of macrophages into osteoclasts. Consistently, Nfatc1 expression was dramatically inhibited by afatinib (Fig. $2 \mathrm{~A}$ and $2 \mathrm{~B})$.

After formation, multinucleated osteoclasts go through an activation stage wherein osteoclasts polarize and develop a specialized membrane structure known as a ruffled border (27). These mature osteoclasts acquire the capacity to resorb bone. As shown in Fig. 3B, mature osteoclasts on the bone slice formed pits on their surface; and afatinib dramatically inhibited pit formation, suggesting that afatinib significantly suppresses the bone resorption activity of mature osteoclasts. The suppressive activities of afatinib on bone resorption as well as osteoclast differentiation may contribute to reducing the osteolytic bone phenotype caused by bone metastasis of NSCLC.

RANK signaling pathways underlying osteoclast differentiation can be modulated by EGFR signaling. This effect was demonstrated using reversible EGFR-TKIs such as AG1478 and PD153035. AG1478 suppressed the RANKL-mediated activation of osteoclastogenic signaling pathways, including JNK, nuclear factor $\kappa B(N F-\kappa B)$, and $A k t / P K B$ (15). Whereas, afatinib inhibited JNK and $A k t / P K B$, it had no effect on $1 \kappa B$ degradation and p65 phosphorylation, indicating no changes in NF- $\mathrm{kB}$ activation. The differential inhibition profile of RANK signaling pathways may be attributed to the covalent binding of afatinib to the EGFR-TK domain. Recently, resistance to EGFR-TKIs has been attributed to NF-кB (28). Pharmacological and genetic inhibition of $I \kappa B$ kinase restored erlotinib sensitivity by accumulating $I \kappa B$ and subsequently activating NF- $\kappa B$ in the H1650 lung cancer cell line (29). However, occurrence of a resistance mechanism to the formation of osteoclast induced by afatinib and erlotinib and the involvement of NF- $\mathrm{KB}$ requires further investigation. Afatinib induced the transient high phosphorylation of ERK and delayed phosphorylation of p38 (Fig. 4). Again, it can be speculated that irreversible covalent binding of afatinib to cysteine 773 could cause conformational changes in the cytoplasmic domain of EGFR and thus may disturb the tyrosine phosphorylation profile, resulting in alterations in signaling pathways.

In summary, afatinib showed an inhibitory effect on osteoclast differentiation of bone marrow monocytes/macrophages and bone resorbing activity of osteoclasts. Afatinib significantly inhibited RANK signaling pathways. Therefore, our results suggested that afatinib relieves the skeletal burden after bone metastasis in particular osteolytic lesions. In addition, because RANKL is a critical factor responsible for osteoclast differentiation and activation in other pathological conditions such as osteoporosis and inflammatory bone erosion, and afatinib inhibited RANKL-induced osteoclast differentiation and activation, afatinib has potential as a therapeutic agent for other skeletal diseases.

\section{MATERIALS AND METHODS}

\section{Mice and reagents}

Six-week-old male ICR mice were purchased from Dae Han Bio Link (Chungbuk, Korea). All animal experiments were approved by the committees on the care and use of animals in research at Kyungpook National University and were conducted in accordance with the guidelines for the care and use of laboratory animals. Recombinant mouse M-CSF and mouse RANKL were obtained from R\&D Systems (Minneapolis, MN, USA). Afatinib (BIBW2992) was purchased from 
Selleckchem, (Houston, TX, USA). Fetal bovine serum (FBS) and $\alpha$-minimum essential medium ( $\alpha$-MEM) were obtained from Gibco BRL (Grand Island, NY, USA).

\section{Osteoclastogenesis}

Bone marrow cells (BMCs) were collected from 6-week-old male ICR mice sacrificed by $\mathrm{CO}_{2}$ inhalation, as previously described (30). BMCs were incubated in $\alpha$-MEM containing $10 \% \mathrm{FBS}$ and M-CSF (30 ng/ml) for 3 days. To generate mature osteoclasts, bone marrow macrophages (BMMs) were plated in 96-well plates and incubated with afatinib $(1,2.5$, and $5 \mu \mathrm{M})$ in the presence of RANKL $(20 \mathrm{ng} / \mathrm{ml})$ and M-CSF $(10 \mathrm{ng} / \mathrm{ml})$.

After 4 days of culture, the cells were fixed and stained with tartrate-resistant acid phosphatase (TRAP)-staining solution prepared following the manufacturer's instructions (SigmaAldrich, St. Louis, MO, USA). TRAP-positive multinucleated cells (MNCs) with $>3$ nuclei were counted using a microscope.

\section{Cell viability assay}

The cell viability of BMMs was measured using the 3(4,5-dimethylthiazol-2-yl)-2,5-diphenyltetrazolium bromide (MTT, Sigma-Aldrich) assay. BMMs were cultured with various concentrations of afatinib in the presence of M-CSF $(10 \mathrm{ng} / \mathrm{ml})$. After incubation for 3 days, MTT was added to each well, and the plate was incubated for $2 \mathrm{~h}$. Absorbance was measured at $570 \mathrm{~nm}$ using a 96-well microplate reader (BioRad, Hercules, CA, USA).

\section{Quantitative real-time PCR}

Total RNA was isolated from cells using the TRI-solution (Bioscience, Seoul, Korea), and the mRNA was reversetranscribed by SuperScript II reverse transcriptase (Invitrogen, Carlsbad, CA, USA). Quantitative real-time PCR was performed in a LightCycler 1.5 real-time PCR system (Roche Diagnostics, Basel, Switzerland) using TOPreal qPCR $2 \times$ PreMIX with SYBR green (Enzynomics, Daejeon, Korea). The primers and conditions used for PCR were as previously described (31).

\section{Western blot analysis}

Total protein was extracted using RIPA buffer containing protease and phosphatase inhibitors. The protein concentration was measured with a BCA protein assay kit (Pierce Biotechnology, Rockford, IL, USA), and equivalent amounts of total protein $(30 \mu \mathrm{g})$ were separated by $10 \%$ sodium dodecyl sulfate polyacrylamide gel electrophoresis. Next, the proteins were transferred to nitrocellulose membranes (Whatman, Florham Park, NJ, USA). After transfer, the membranes were incubated with $3 \%$ non-fat dry milk in TBS-T $(25 \mathrm{mM}$ Tris- $\mathrm{HCl}, \mathrm{pH} 7.4,150 \mathrm{mM} \mathrm{NaCl}$, and $0.2 \%$ Tween 20) to block nonspecific binding sites. The membranes were incubated overnight with primary antibodies at $4^{\circ} \mathrm{C}$, followed by incubation with the appropriate secondary antibodies. Proteins were detected using the WesternBright enhanced chemilumi- nescent substrate (Advansta, Menlo Park, CA, USA). Specific antibodies against phospho-p38, phospho-JNK, phospho-ERK, phospho-AKT, phospho-p65, and phospho-I $\mathrm{KB} \alpha$ were purchased from Cell Signaling Technology (Danvers, MA). Monoclonal anti- $\beta$-actin was obtained from Sigma-Aldrich (St. Louis, MO).

\section{Immunofluorescence}

BMMs were cultured on glass coverslips with RANKL (20 $\mathrm{ng} / \mathrm{ml})$ and M-CSF $(10 \mathrm{ng} / \mathrm{ml})$ either in the presence or absence of $2.5 \mu \mathrm{M}$ afatinib for 4 days. The cells were fixed with $4 \%$ paraformaldehyde, permeabilized using $0.25 \%$ Triton X-100, followed by blocking in blocking buffer ( $3 \%$ bovine serum albumin in PBS) for $1 \mathrm{~h}$. The cells were incubated with an anti-NFATc1 antibody, followed by incubation with an Alexa Fluor-488 conjugated secondary antibody (Invitrogen, Carlsbad, CA, USA). F-actin was stained with rhodamine-conjugated phalloidin (Cytoskeleton, Denver, CO, USA) and nuclei with 4',6-diamidino-2-phenylindole dihydrochloride (DAPI; Santa Cruz Biotechnology, Santa Cruz, CA, USA). Fluorescent images were obtained using a BX51 fluorescence microscope (Olympus, Tokyo, Japan).

\section{Resorption pit assay}

Mouse BMMs were seeded on bone slices (IDS Nordic, Herlev, Denmark) and cultured with M-CSF (10 ng/ml) and RANKL $(20 \mathrm{ng} / \mathrm{ml})$ for 3 days to induce osteoclast differentiation. Subsequently, the cells were incubated with or without afatinib $(2.5 \mu \mathrm{M})$ for 2 days. All cells were removed from the bone slices, and Mayer's hematoxylin staining was used to visualize the pit area. The area of resorbed pits was measured using the i-Solution image analysis software (IMT i-Solution, Daejeon, Korea).

\section{Statistical analyses}

All experiments were conducted three times, and the data are presented as the mean \pm standard deviation (SD). Statistical analyses were performed by the two-tailed Student's t-test or one-way analysis of variance with Tukey's multiple comparison post-hoc test. A $\mathrm{P}$ value of $<0.05$ was considered statistically significant.

\section{ACKNOWLEDGEMENTS}

This work was supported by grants from the National Research Foundation of Korea (NRF) funded by the Ministry of Science, ICT, and Future Planning (MSIP-2008-0062282 and NRF2016R1A6A3A01006911).

\section{CONFLICTS OF INTEREST}

The authors have no conflicting financial interests. 


\section{REFERENCES}

1. Asagiri $M$ and Takayanagi $H$ (2007) The molecular understanding of osteoclast differentiation. Bone 40, 251-264

2. Teitelbaum SL and Ross FP (2003) Genetic regulation of osteoclast development and function. Nat Rev Genet 4, 638-649

3. Dougall WC, Glaccum M, Charrier K et al (1999) RANK is essential for osteoclast and lymph node development. Genes Dev 13, 2412-2424

4. Kong YY, Yoshida H, Sarosi I et al (1999) OPGL is a key regulator of osteoclastogenesis, lymphocyte development and lymph-node organogenesis. Nature 397, 315-323

5. Hirsh V, Major PP, Lipton A et al (2008) Zoledronic acid and survival in patients with metastatic bone disease from lung cancer and elevated markers of osteoclast activity. J Thorac Oncol 3, 228-236

6. D'Antonio C, Passaro A, Gori B et al (2014) Bone and brain metastasis in lung cancer: recent advances in therapeutic strategies. Ther Adv Med Oncol 6, 101-114

7. Mourskaia AA, Dong Z, Ng S et al (2009) Transforming growth factor-beta1 is the predominant isoform required for breast cancer cell outgrowth in bone. Oncogene 28, 1005-1015

8. Rossi A, Gridelli C, Ricciardi S and de Marinis F (2012) Bone metastases and non-small cell lung cancer: from bisphosphonates to targeted therapy. Curr Med Chem 19, 5524-5535

9. Silva SC, Wilson C and Woll PJ (2015) Bone-targeted agents in the treatment of lung cancer. Ther Adv Med Oncol 7, 219-228

10. Maemondo M, Inoue A, Kobayashi K et al (2010) Gefitinib or chemotherapy for non-small-cell lung cancer with mutated EGFR. N Engl J Med 362, 2380-2388

11. Zhou C, Wu YL, Chen G et al (2011) Erlotinib versus chemotherapy as first-line treatment for patients with advanced EGFR mutation-positive non-small-cell lung cancer (OPTIMAL, CTONG-0802): a multicentre, openlabel, randomised, phase 3 study. Lancet Oncol 12, 735-742

12. Kosaka T, Yatabe $Y$, Endoh $\mathrm{H}$ et al (2006) Analysis of epidermal growth factor receptor gene mutation in patients with non-small cell lung cancer and acquired resistance to gefitinib. Clin Cancer Res 12, 5764-5769

13. Wind S, Schnell D, Ebner T, Freiwald $M$ and Stopfer $P$ (2017) Clinical Pharmacokinetics and Pharmacodynamics of Afatinib. Clin Pharmacokinet 56, 235-250

14. Wang K, Yamamoto $\mathrm{H}$, Chin JR, Werb Z and Vu TH (2004) Epidermal growth factor receptor-deficient mice have delayed primary endochondral ossification because of defective osteoclast recruitment. J Biol Chem 279, 53848-53856

15. Yi T, Lee HL, Cha JH et al (2008) Epidermal growth factor receptor regulates osteoclast differentiation and survival through cross-talking with RANK signaling. J Cell Physiol $217,409-422$
16. Miyauchi $\mathrm{Y}$, Ninomiya K, Miyamoto H et al (2010) The Blimp1-Bcl6 axis is critical to regulate osteoclast differentiation and bone homeostasis. J Exp Med 207, 751-762

17. Zhao B, Takami M, Yamada A et al (2009) Interferon regulatory factor- 8 regulates bone metabolism by suppressing osteoclastogenesis. Nat Med 15, 1066-1071

18. Jurdic P, Saltel F, Chabadel A and Destaing O (2006) Podosome and sealing zone: specificity of the osteoclast model. Eur J Cell Biol 85, 195-202

19. Teitelbaum SL (2007) Osteoclasts: what do they do and how do they do it? Am J Pathol 170, 427-435

20. Lynch TJ, Bell DW, Sordella R et al (2004) Activating mutations in the epidermal growth factor receptor underlying responsiveness of non-small-cell lung cancer to gefitinib. N Engl J Med 350, 2129-2139

21. Paez JG, Janne PA, Lee JC et al (2004) EGFR mutations in lung cancer: correlation with clinical response to gefitinib therapy. Science 304, 1497-1500

22. Li D, Ambrogio L, Shimamura T et al (2008) BIBW2992, an irreversible EGFR/HER2 inhibitor highly effective in preclinical lung cancer models. Oncogene 27, 4702-4711

23. Solca F, Dahl G, Zoephel A et al (2012) Target binding properties and cellular activity of afatinib (BIBW 2992), an irreversible ErbB family blocker. J Pharmacol Exp Ther 343, 342-350

24. Modjtahedi H, Cho BC, Michel MC and Solca F (2014) A comprehensive review of the preclinical efficacy profile of the ErbB family blocker afatinib in cancer. Naunyn Schmiedebergs Arch Pharmacol 387, 505-521

25. Normanno N, De Luca A, Aldinucci D et al (2005) Gefitinib inhibits the ability of human bone marrow stromal cells to induce osteoclast differentiation: implications for the pathogenesis and treatment of bone metastasis. Endocr Relat Cancer 12, 471-482

26. Furugaki K, Moriya Y, Iwai T et al (2011) Erlotinib inhibits osteolytic bone invasion of human non-small-cell lung cancer cell line NCl-H292. Clin Exp Metastasis 28, 649659

27. Itzstein C, Coxon FP and Rogers MJ (2011) The regulation of osteoclast function and bone resorption by small GTPases. Small GTPases 2, 117-130

28. Shostak K and Chariot A (2015) EGFR and NF-kappaB: partners in cancer. Trends Mol Med 21, 385-393

29. Bivona TG, Hieronymus $\mathrm{H}$, Parker J et al (2011) FAS and NF-kappaB signalling modulate dependence of lung cancers on mutant EGFR. Nature 471, 523-526

30. Ihn HJ, Lee D, Lee T et al (2015) The 1,2,3-triazole derivative KP-A021 suppresses osteoclast differentiation and function by inhibiting RANKL-mediated MEK-ERK signaling pathway. Exp Biol Med (Maywood) 240, 16901697

31. Ihn HJ, Lee D, Lee $T$ et al (2015) Inhibitory Effects of KP-A159, a Thiazolopyridine Derivative, on Osteoclast Differentiation, Function, and Inflammatory Bone Loss via Suppression of RANKL-Induced MAP Kinase Signaling Pathway. PLoS One 10, e0142201 